\title{
Postponement Based on the Positioning of the Differentiation and Decoupling Points
}

\author{
Joakim Wikner ${ }^{1}$ and Hartanto Wong ${ }^{2}$ \\ 1 Jönköping University, School of Engineering, \\ Jönköping, S-551 11, Sweden, joakim.wikner@jth.hj.se \\ 2 Cardiff University, Innovative Manufacturing Research Centre \\ Colum Drive, Cardiff CF10 3EU, Wales-UK, wongh@Cardiff.ac.uk
}

\begin{abstract}
Structural analysis of supply chains often involves postponement to some degree. The concept has received a lot of attention in the literature but the understanding of how to operationalize the concept still deserves some attention. A framework is introduced that provides an integrative picture of how different aspects of supply chain and operations management interplay in a postponement context. The framework is founded on a process/object perspective where a set of characteristics, properties and concepts are identified for each entity.
\end{abstract}

\section{Introduction}

Postponement or delayed product differentiation is an important concept used to accommodate mass customization that has been increasingly receiving attention from researchers and practitioners. It is an effective way to manage the risks associated with proliferating product variety without incurring large operating costs. Although the concept has been around for years, our literature review suggests that there is still a lack of understanding of how the concept is operationalized. Several authors have introduced different conceptual categorization of postponement strategies extending the understanding of where and when postponement is appropriate (see e.g. [1] among others for a comprehensive literature review of postponement).

Despite their differences, most conceptual classifications found in the literature share a commonality in that they all refer to postponement as the delaying of certain operations until customer orders are received. Such a concept suggests that the final 
differentiation process needs to be performed in a make-to-order (MTO) fashion. Ideally, this concept would maximize the profits of postponement as it omits the inventory of the final products. However, it is obvious that in reality it may not always be possible to employ such a postponement strategy especially in the highly responsive environments where the tolerance time that the customer is willing to wait is quite short. In such environments it may be necessary to produce the final products in a make-to-stock (MTS) fashion. From that perspective, we argue that the concept referring to postponement as the delaying of activities until customer orders are received does not always represent the best course of action. This motivated us to undertake a study looking at a more complete set of manufacturing configurations related to the implementation of postponement strategy.

\section{Key entities in postponement analysis}

Postponement plays an important role in operations and supply chain management as it captures both the issue of where in the supply differentiation takes place and where decoupling points play a strategic role. As a foundation for investigating these different perspectives we have adopted a process/object perspective when developing this framework. In addition to the process we have highlighted three different types of objects of which two are discussed further in the sub-sections below. In summary, the framework focuses on:

- Customer objects, formulating the requirements.

- Product objects, having a level of customization to fulfil customer requirements.

- Resource objects, providing a level of flexibility to enable customization.

- Processes, embracing the level of uncertainty by exploiting resource flexibility to fulfil customer requirements.

\subsection{Product object modelling}

It can be argued that the increasing pressure to become more and more customercentric has forced manufacturing firms to formulate their marketing and operations strategies around mass customization [2]. Mass customization has been defined as the technologies and systems to deliver goods that meet individual customers' needs with near mass production efficiency. A quintessential feature of mass customization is the proliferation of product variety. The prevailing view is that offering larger variety allows firms to increase both demand and market share [3]. However, it has also been recognized that a large number of product variety is associated with diseconomies of scale and increases in production and distribution costs. Postponement, also termed as delayed product differentiation or late customization represents a way to implement mass customization without incurring large operating costs associated with managing proliferating product variety. This is done by properly designing the product structure and the manufacturing and supply chain process so that one can delay the point in which the final customization of the product is to be configured. Fig. 2 and Fig. 3 contrast two different configurations that are different from each other in terms of the position of the differentiation point. 
The first configuration (Fig. 1) is the single-stage system where end product configurations (PCs) are processed and customized through a single-stage production originating in raw materials (RM). The second system employing postponement consists of two stages. Stage 1 produces the generic components and Stage 2 differentiates the final product configurations.

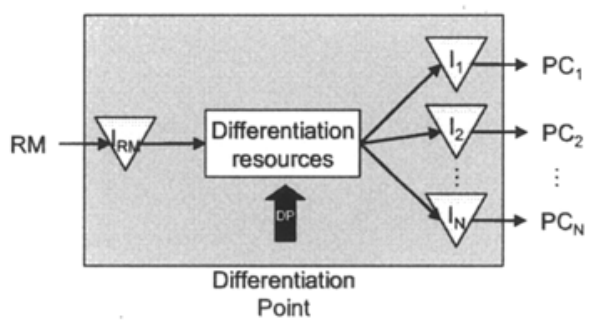

Fig. 1. Single-stage early differentiation

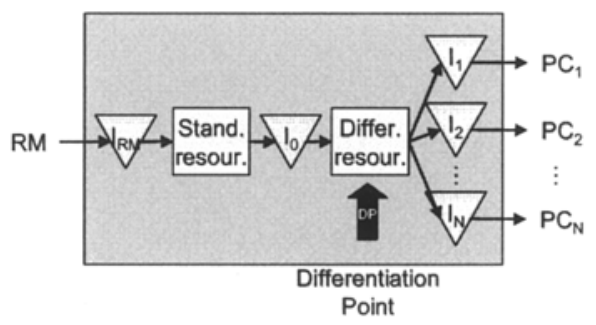

Fig. 2. Two-stage delayed differentiation

\subsection{Resource object modelling}

Our focus of the resource object modelling is on the flexibility, which is an important resource characteristic when dealing with the proliferation of product variety. Flexible manufacturing systems (FMS) allow a firm to cope better with demand uncertainty when manufacturing several products in the same facility. This also relates to the well-known result in the queuing systems literature specifying that employing a flexible resource (Fig. 4) instead of several dedicated resources (Fig. 3) may offer so called pooling benefits resulting in improved system's performances such as reduced expected waiting times in the queue. These benefits, however, could be distorted by the presence of non-negligible changeover times. Diseconomies of scope associated with the increase of product variety are particularly true for manufacturing environments with significant changeover times. In the presence of significant changeover times products are usually manufactured in batches to achieve economies of scale, which would in turn lengthen the manufacturing lead times and consequently increase the inventory and backorder levels. This unfavorable effect will be more pronounced whenever the system produces more product configurations. 


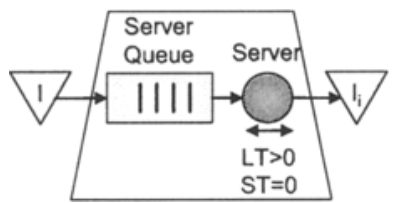

Fig. 3. Dedicated resource

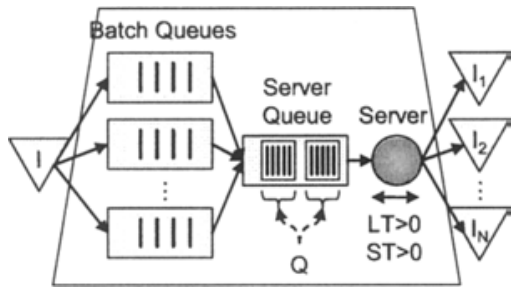

Fig. 4. Flexible resource

Four different configurations, as shown in Fig. 5, emanate from combining product and resource objects.
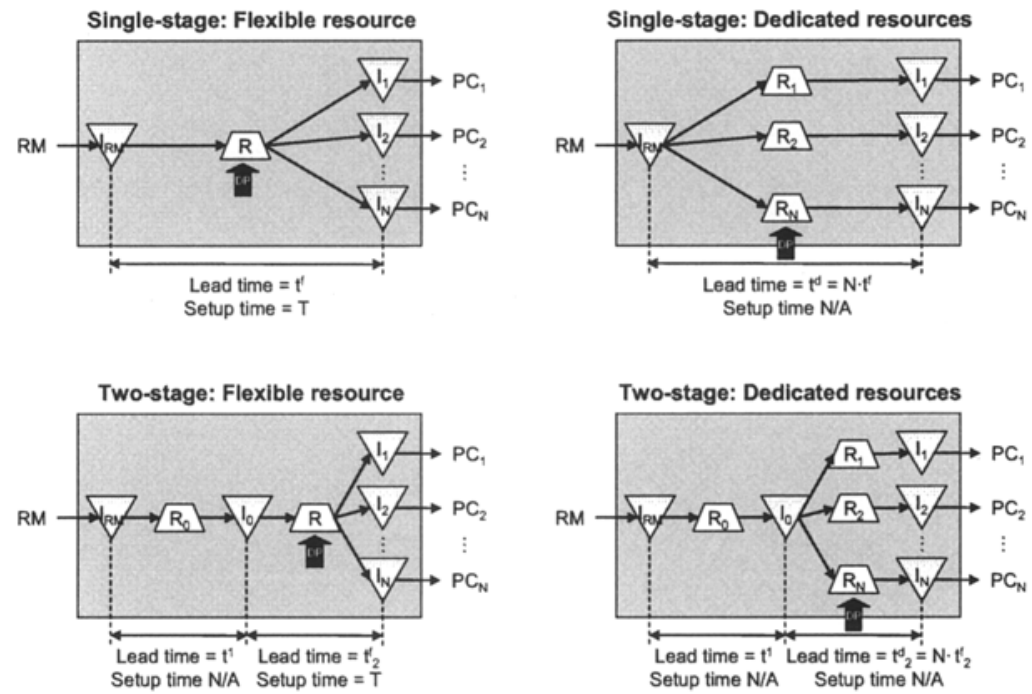

Fig. 5. Product-resource combinations

It is worth noting that from a system perspective, common material (generic component) used in the configurations employing postponement (the two-stage configurations) can also be seen as an analogy to a flexible resource with no changeover time being required when switching production from one product to another. The main difference is that the resources stays in that state during production of a batch and then is set up for a new assignment whereas the common material enters a product and then usually stays in that state during the rest of its life 
cycle. But when deciding on how to use the object the time from decision to operation does not require any delay for set up as the resource/material is flexible.

\subsection{Process modeling}

Fisher [4] proposed a model for the matching of product characteristics and supply chain design. A supply chain has two generic functions. First, it has a physical function of transforming physical goods promoting an efficient flow of products with high resource utilisation. Second, a supply chain has a market mediating function of conveying information from the point of sales to upstream of the supply chain, promoting quick response to variations in customer demand and a high level of flexibility through the supply chain. The focus of the supply chain in terms of physical efficiency and market mediation will affect the way the supply chain is designed. In relation to this, we highlight an important concept that relates to the position of the buffer usually referred to as a decoupling point.

Two decoupling points are of particular interest [6]. The first is the physical supply decoupling point (PSDP) also called the customer order decoupling point (CODP) or order penetration point (OPP) [5]. At a more detailed level the process can be characterized as supporting different product delivery strategies based on the position of the CODP. The second decoupling point is called the demand mediation decoupling point (DMDP), which is not related to a buffer in the conventional physical sense but to a decoupling point from an information perspective. The DMDP simply indicates where demand information is available but provides no direction for how the information is supposed to be used in the physical supply of products [6]. Combining the two decoupling points result in a decoupling point framework illustrated in Fig. 6. In Fig. 7 we show the demand mediation process for the flexible and dedicated resources.

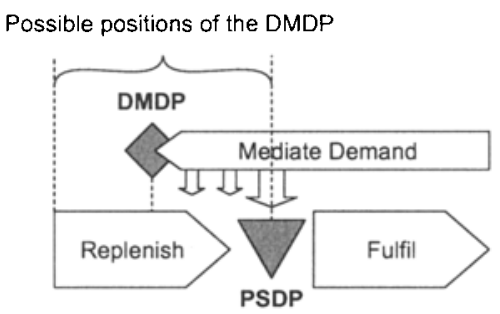

Fig. 6. Process configuration framework [6]. 


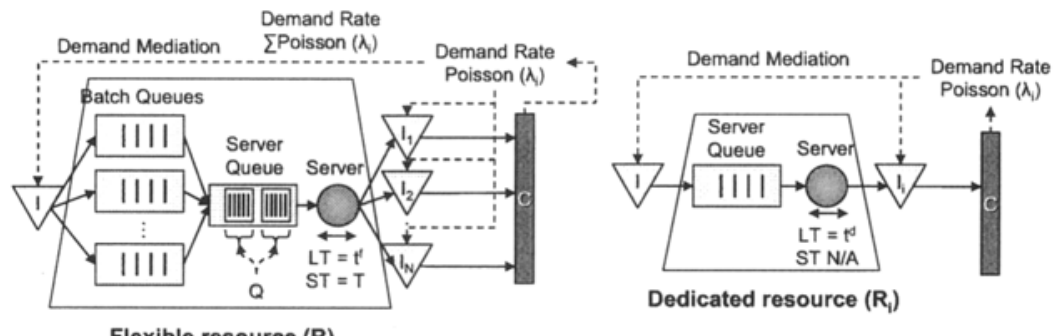

Flexible resource $(R)$

Fig. 7. Demand mediation and resources.

A more complex approach would allow the possibility to produce some of the product configurations to stock and others to order resulting in a wide range of possible system configurations. Some examples of possible configurations are shown in Fig. 8 for the case of two-stage flow with flexible resource at stage 2 . Since there are three possible positions of the CODP/OPP decoupling buffers (at OP in Fig. 8) there are three possible configurations. In total there are 10 possible configurations considering the differentiation point, the type of resource configuration and the positioning of the CODP/OPP (see Table 1).
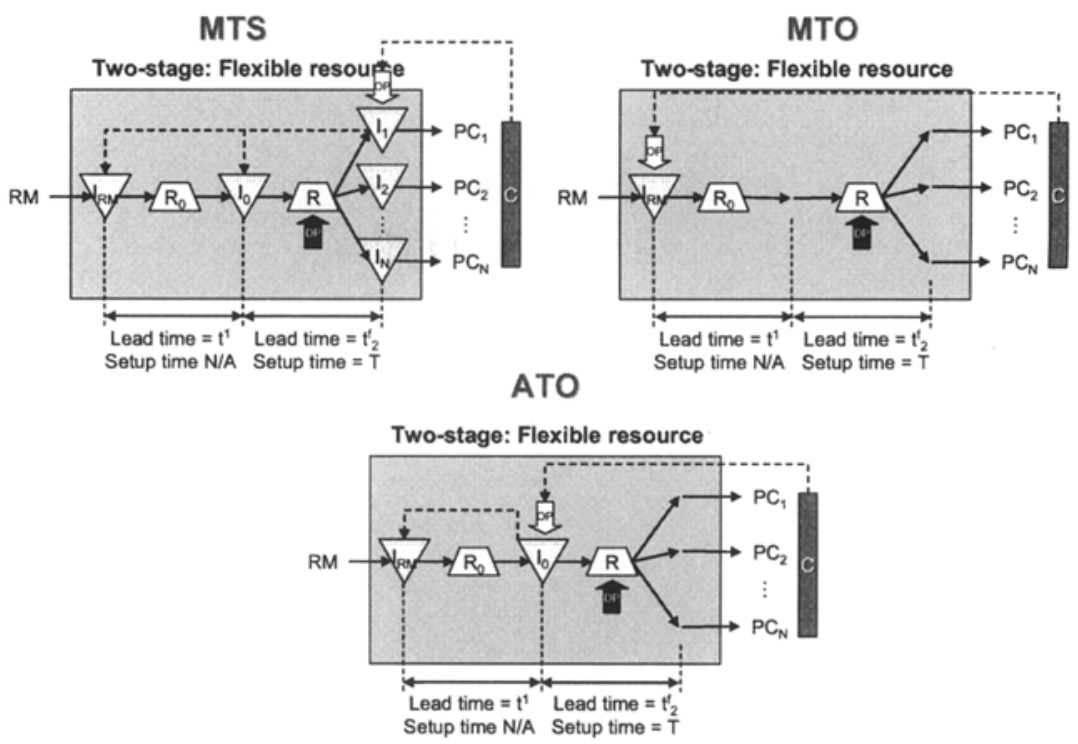

Fig. 8. Example of system configurations with different OPP/CODP 
Table 1. Number of possible positions of the CODP/OPP

\begin{tabular}{|l|c|c|}
\hline & Single-stage flow & Two-stage flow \\
\hline Flexible resource & 2 & 3 \\
\hline Dedicated resource & 2 & 3 \\
\hline
\end{tabular}

\section{Some findings}

In [7-8] we developed analytical models and carried out numerical experiments for making the evaluation of different system configurations. Some important findings are highlighted in the following.

- Delaying product differentiation in postponement does not necessarily suggest that the differentiation is only processed after customer orders are received. Different system parameters have an impact on the choice of optimal system configuration.

- Our numerical results confirm that the diseconomies of scope associated with the increase of product variety occur even though the total volume of demand does not change. The total costs increase very rapidly as the changeovers take longer. It becomes obvious that the marketing strategy, which is usually in favour of offering more product variants to achieve a higher degree of customization must be aligned with the manufacturing strategy in order to minimize costs or maximize profits.

- Sharing a common material as allowed by postponing product differentiation i.e. moving from a single-stage system to a two-stage system, is always beneficial while sharing a common production resource may be beneficial but may also be disadvantageous depending on the changeover time incurred. It is however important to note that there is usually a premium cost associated with redesigning the product to accommodate material commonality that needs to be balanced against the benefits of postponement.

- The presence of significant changeover times associated with the use of a flexible resource reduces the possibility to employ the most desirable system configuration where the CODP/OPP is positioned far enough upstream allowing final products are made with certain demand. This observation suggests that continuous efforts in reducing changeover times are essential for maximizing the benefits of postponement.

\section{Conclusion}

Postponement is an important concept in operations and supply chain management as it highlights the strategic importance of the positioning of differentiation and decoupling points. These aspects are however not usually discussed from a modelling perspective highlighting the key constructs used in process modelling. In this paper we have introduced a postponement framework based on products, resources and processes. The framework highlights and positions 
a set of characteristics, properties and concepts in response to customer requirements for different levels of customization, as summarized below.

- Product Objects

- Key product characteristic: Level of customization

- Key customization property: Number of variants

- Important concepts: Differentiation point and commonality

- Resource Objects

- Key resource characteristic: Level of flexibility

- Key flexibility property: Volume and mix flexibility

- Important concepts: Changeover time and capacity

- Process

- Key process characteristic: Level of uncertainty

- Key uncertainty property: Demand uncertainty in process driver

- Important concepts: Physical supply decoupling point (CODP/OPP) and Demand mediation decoupling point

In summary, these three entities and their respective attributes in terms of characteristics, properties and concepts provides a framework that supports the operationalization of postponement by enabling an integrative perspective of postponement from an object/process perspective.

\section{References}

1. R.I. Van Hoek, The rediscovery of postponement: a literature review and directions for research, Journal of Operations Management 19, 161-184 (2001).

2. M. Agrawal, T.V. Kumaresh, and G.A. Mercer, The false promise of mass customization, The McKinsey Quarterly 3, 62-71 (2001).

3. P. Kotler, Marketing Management (Prentice Hall, 2002).

4. M. Fisher, What is the right supply chain for your product?, Harvard Business Review 75(2), 105-116 (1997).

5. G. Sharman, The rediscovery of logistics, Harvard Business Review 62(5), 71-80 (1984).

6. J. Olhager, E. Seldin, and J. Wikner, Decoupling the value chain, International Journal of Value Chain Management 1(1), 19-32 (2006).

7. H. Wong, J. Wikner, and M. Naim, Analysis of form postponement based on optimal positioning of the differentiation point and stocking decisions, Working Paper, Cardiff Business School, 2006 (unpublished).

8. H. Wong, J. Wikner, and M. Naim, Evaluation of postponement in manufacturing environments with non-negligible changeover times, Working Paper, Cardiff Business School, 2007 (unpublished). 